\title{
An Efficient Algorithm for Shortest Situation Aware Emergency Navigation with WSN
}

\author{
R. Bharathi ${ }^{1}$, Dr. C. Kalaiarasan ${ }^{2}$ \\ PG Scholar, Department of Computer Science and Engineering, Coimbatore, TN, India ${ }^{1}$ \\ Dean, Department of Computer Science and Engineering, Coimbatore, TN, India ${ }^{2}$
}

\begin{abstract}
Large number of sensors in an Emergency navigation can serve as a safety service in emergencies. Recent studies have focused on navigation protocols to safely guide people to exits while helping them avoid hazardous areas. Recently, many applications in wireless sensor networks (WSNs) have been discussed. Navigation with WSNs is the most heated debated ones. Existing navigation algorithms are used to find safe and efficient escape paths for individuals under various environmental dynamics while ignoring possible congestion, cause the individuals rushing for the exits. Previous routing mechanisms for WSN are not well suited for building fires, especially they do not consider critical and dynamic network scenarios. The propose an efficient Shortest Situation-Aware Emergency Navigation Algorithm based ant colony path selection by treating WSNs as navigation infrastructure. It takes the hazard levels of emergencies and the evacuation capabilities of exits into an account and provides the mobile users for the safest navigation paths accordingly. We formally model the situation-aware emergency navigation problem and establish a hazard potential field in the network, which is theoretically free of local minima. It was performed by extensive simulations under WSN environments to evaluate the effectiveness and response time of our work. Simulation result shows that, with our strategy, people in emergency are evacuated by less congestion and much faster to exit is observed.
\end{abstract}

Keywords: Wireless sensor network; Emergency Saving Navigation system; Ant Colony Optimization Algorithm.

\section{INTRODUCTION}

The navigation problem is often used for trajectory planning for planes and moving planning for robots. Navigation is the process of monitoring and controlling the movement of a craft or vehicle from one place to another. In general, navigation is seen as a service which prevents the mobile user (e.g., robots, aircrafts or other people) from moving into undesirable areas (e.g., walls, obstacles or roadblocks) or dangerous areas (e.g., fire, or contaminated areas). Most existing movement planning algorithms based on combinatorial optimization, computational geometry, and graph theory are centralized and assume the availability of global knowledge about the path topology. For example, widely used road navigation applications in Global Positioning Systems (GPS) use centralized path planning algorithms with pre-loaded maps of the navigation environment.

A real-time variable safety net could be made of hundreds of small sensors with limited memory and multiple sensing capabilities that autonomously organize and reorganize themselves as ad-hoc networks in response to emergency tasks and to triggers from the environment. When an emergency happens, the navigation system deployed in advance is triggered to implement two guiding tasks. In the first, a trapped user needs to be guided out of the emergency area avoiding dangerous areas. In the second, rescuers need to be guided into the emergency region to help trapped people in danger.

In the near future, it can be expected that buildings will be equipped with a range of wireless sensors functioning as part of an overall building management system.

Included in this set of sensors will be devices to monitor fire and smoke, allowing detection, localization and tracking of fires. It is expected such information could be used for a variety of purposes, including guiding building occupants to the nearest safe exit, and helping firefighting personnel to decide on how to best tackle the disaster. Fire/smoke sensors are expected to be programmed to report periodically and also when they detect a sensor input that exceeds a threshold. In the latter case, there is a need for an emergency-adaptive, real-time and robust message delivery toward the sink. For example, a fire-fighter relies on timely temperature updates to remain aware of current fire conditions. In addition, as the fire spreads throughout the building, it becomes likely that the sensing devices may become disconnected from the network or indeed be destroyed, so the network routes have to be changed or re-discovered to adapt to these emergency conditions in order for the network to continue operating. Most existing routing protocols consider the energy efficiency and lifetime of the networks as the foremost design factor. 
Navigation algorithms proposed previously search for the shortest or the safest path and a self-organizing sensornetwork is proposed to guide a user (robots, people, unmanned aerial vehicle, etc.) through the safest path by using "artificialpotential fields". An attractive force pulls users to the destination while repulsive forces from dangerous zones pushthem away. In a temporally ordered routing algorithm routes evacuees to exits through safer paths. A navigation mapis manually defined to avoid impractical paths and each sensor is assigned with an altitude with respect to itshops to thenearliest exit. Combining the definition of effective length with Dijkstra's algorithm, presents a decentralized evacuationsystem with decision nodes (DNs) and sensor nodes (SNs) to compute the shortest routes in real time, while opportunisticcommunications based emergency evacuation systems have the advantage of being more robust to networkattacks that often accompany emergencies caused by malicious acts.

\section{RELATED WORK}

The first algorithm, named Lloyd- $\alpha$, reduces the movement step sizes in Lloyd 's method. It saves travelling distance while maintaining the convergence property. However, it leads to a larger number of deployment steps. The second algorithm, named DEED (Distributed Energy-Efficient Self-Deployment), reduces sensor travelling distances and requires a comparable number of deployment steps as that in Lloyd 's method. This paper further proposes an intuitive method to deal with limited sensor communication range that is applicable to all three methods

Many firefighters are trained to search a dark room while keeping either their left or right hand in contact with the wall, which helps with orientation and provides a strategy for systematically exploring an unknown space. Human contact and accountability are also essential. Fire fighters always perform searches in teams of at least 2.8 During a lifeline search, one team member might stay at a fixed position to help with orientation and provide progress reports to the commander while other colleagues are engaged in a deeper search. Team members report their locations as accurately as possible over the radio to a commander posted outside the building; this person often keeps track of team locations on a whiteboard.

The reactive task in sensor networks: guiding the movement of a user equipped with a node that can talk to the field of sensors across the field. We also discuss how sensor networks can serve as adaptive distributed repositories of information. Current work in reactive routing protocols (or directed diffusion) are aiming for the network communication. They cannot provide the navigation information to the user in the sensor field. We combine robotics and networking. We model the user guidance problem as a robot motion planning problem and use the inherent feature of the sensor network to compute the robot navigation path in a distributed way. Our paper contributes: (1) an interesting application for sensor network; (2) an implementation and evaluation on a physical sensor network; (3) a distance computation method that does not use node positions; (4) performance analysis and hardware experimentation. In this work, we consider the joint problem of mobile sensor navigation and mobile target tracking based on a TOA measurement model. Our chief contributions include a more general TOA measurement model that accounts for the measurement noise due to multipath propagation and sensing error. Based on the model, we propose a min-max approximation approach to estimate the location for tracking that can be efficiently and effectively solved by means of semidefinite programming (SDP) relaxation. We apply the cubic function for navigating the movements of mobile sensors. In addition, we also investigatethe simultaneous localization of the mobile sensor and the target to improve the tracking accuracy. We present a weighted tracking algorithm in order to exploit the measurement information more efficiently. The numerical result shows that the proposed tracking approach works well.

The scalable solutions for the problem of navigating a user in the presence of disruptions or hazards in a sensor field. Our algorithms make two natural assumptions: (1) the operational environment is assumed to have no large holes in the coverage by sensors, and (2) an approximately optimal safe path is acceptable. Based on these two assumptions, we develop distributed navigation algorithms that are very efficient in terms of their communication cost; they find nearoptimal paths with significantly smaller communication (and, thus, energy) overhead. The problem of guiding mobile sensors (or robots) to a phenomenon across a region covered by static sensors. We present a distributed, reliable and energy efficient algorithm to construct a smoothed moving trajectory for a mobile robot. The reliable trajectory is realized by first constructing among static sensors a distributed hop count based artificial potential field (DH-APF) with only one local minimum near the phenomenon, and then navigating the robot to that minimum by an attractive force following the reversed gradient of the constructed field. Besides the attractive force towards the phenomenon, our algorithm adopts an additional repulsive force to push the robot away from obstacles, exploiting the fast sensing devices carried by the robot. Compared with previous navigation algorithms that guide the robot along a planned path, our algorithm can (1) tolerate the potential deviation from a planned path, since the DH-APF covers the entire deployment region; (2) mitigate the trajectory oscillation problem; (3) avoid the potential collision with obstacles; (4) save the precious energy of static sensors by configuring a large moving step size, which is not possible for algorithms neglecting the issue of navigation reliability

The integrate the information of dangerous areas and users to provide a best effort emergency navigation schedule. Therefore, we propose SOS (Safe, Ordered, and Speedy) emergency navigation algorithm. SOS can compute an 
optimal. Preliminary simulations show the proposed framework is able to solve path planning problem with low communication overhead. In this paper, we propose a distributed planning framework, Distributed PRM (D-PRM), to systematically incorporate a general spatial sensing model for each sensor. It takes into account "obstacles" in determining feasible paths. Online method to explicitly compute a set of homotopically different paths to the goal, i.e., a set of paths that cannot be smoothly transformed into each other without colliding with obstacles. Trajectory optimization approaches can benefit from parallel initialization with these paths from multiple homology classes by selecting the best result after individual optimization. In addition, instead of discarding the remaining optimized trajectories after selecting the best one, it can also be beneficial to maintain a set of optimized homotopically different trajectories during navigation. While during one packetdelivery process, the network is often considered static,human navigation in contrast deals with emergencydynamics almost all along theguiding process. As such, wecannot simply borrow existing packet routing schemes foremergency navigation with WSNs.

\section{PROPOSED APPROACH}

The presentshortest situationaware emergency navigation algorithm, which takes the hazard levels of emergencies and theevacuation capabilities of exits into account and providesthe mobile users the safest navigation paths accordingly.Motivated by the fact that the natural gradients of somephysical quantities always follow a natural diffusion law,e.g., water always flows from the place with a higher gravitypotential to that with a lower gravity potential, we thuspropose to model the hazard levels of emergencies and theevacuation capabilities of exits as hazard potentials withpositive and negative values, respectively. Then we establisha hazard potential field in the network, which is theoreticallyfree of local minima. By guiding users following thedescend gradient of the hazard potential field, our methodcan thereby achieve guaranteed success of navigation andprovide optimal safety to users. The first shortest situationawareemergency navigation scheme, considering the impactsof both the hazard levels of emergencies and theevacuation capabilities of exits. It is fully distributed anddoes not require any location information. It is more robustto emergency dynamics since the constructed hazard potentialfield reflects more global properties of the underlyingconnectivity. Both small-scale testbed experiments and extensivesimulations on large-scale WSNs.

\section{A. Network Model}

Network model consider a field where there may be different emergency events and multiple exits with different evacuation capabilities. People inside the field are anticipated to be immediately navigated to appropriate exits while being far away from emergencies in proportion to corresponding hazard levels. Specifically, the emergency navigation paths are expected to be farther away from areas with higher hazard levels, and more people should be guided to exits with higher evacuation capabilities. On the basis of these observations, we thus formulate the navigation problem as a path planning problem.

\section{B. Hazard Point}

An emergency navigation problem is essentially to find the optimal emergency navigation paths in terms of safety. Quantifying the safety of a path is equal to quantifying the hazard of a path, which is closely related to emergency. In the following, we first focus on the hazard of an arbitrary point in the field of interest, which is the basis of finding the safest navigation path. The quantify the hazard of a location, we introduce a novel metric called hazard intensity, which is based on the observation that for an internal user, one may feel more hazardous threat when getting closer to emergencies, and would feel safer when getting closer to exits. Furthermore, the feeling should be a vector that has the ability to describe the direction of the hazardous event. The hazard intensity reflects only an instant feeling of the user, which is not enough to quantify the hazard of a single point. To this end, we introduce a function called hazardous potential, which represents the total hazardous intensity one user has starting from infinityand ending at point $\mathrm{p}$. We choose infinity as a common reference point to evaluate the hazard potential in different spaces

\section{Path Hazard Metric and the Safest Path}

The interested in finding the safest path from an arbitrarypoint to an appropriate exit. The primarychallenge of such problem is to choose the safest path amonghuge amounts of paths, which start from $\mathrm{p}$ and end.So, in the first step, we have to design a path hazard metric toquantify the hazard of a path. As we discussed before, thelocation of point $p$ is more harmful to the user than that ofpoint $q$ if $(p)>(q)$. Intuitively, the hazard of a pathcan be quantitatively measured as the maximum hazardpotential of the points on the path.

\section{IV.SHORTEST SITUATION AWARE EMERGENCY NAVIGATION ALGORITHM}

Based on the aforementioned theoretical foundation in continuousdomains, in this section, we further describe thedetails of algorithm in discrete sensor networks. Wefirst define hazard potential field in the network, which isthe 
discrete counterpart of hazard potential field in continuousdomains. Then we propose an iterative method toestablish the hazard potential field by sensor readings in afully distributed manner. Based on the established hazardpotential field, we next propose a path selection methodand theoretically prove that the selected paths guarantee.

To estimate the speed of hazard, we have to find out thespread distance and the corresponding time. However, it isquite challenging to obtain the distance and time distributedin a WSN-assistant navigation algorithm. Estimatingthe distance may need pre-knowledge of sensors locationinformation as well as two or more sensors exchanging theirreadings, which may incur excessive communication costs, while obtaining the time may require relatively accuratetime synchronization, which may rely on special hardwareor time synchronization algorithms. These requirements arenot suitable for resource-constrained WSNs and greatlyhinder the designed ant colony algorithms from being distributed andlightweight. Thus, theoretically modeling the hazard speedin WSN-assistant navigation itself is still an open problem,and asks for thorough and intensive research.Therefore, existing navigation approaches mainly focus onthe design of a navigation protocol, and deal with hazards Spread by incorporating a supplementary module in themanner of rebuilding the navigation architecture.

The optimization quantity is the distance of the route.Thus, the truck movement cost between loading spots $i$ andj is a function of all separate costs for each factor whichaffects the track route. Each ant at time $t$ chooses the next node, where it willbe at time $t+1$. Therefore, if we call an iteration of the ACO algorithm the $n$ moves carried out by the $n$ ants in theinterval $(t, t+1)$, then for every $n$ iterations of the algorithm,each ant has completed a tour.The coefficient $\rho$ must be set to a value $<1$ to avoidunlimited accumulation of trail (see note 1). In our experiments, we set the intensity of trail at time $0, \mathrm{t}_{\mathrm{ij}}(0)$, to a smallpositive constant c.In order to satisfy the constraint that an ant visits all then differentloading spots, we associate with each ant a datastructure called the hlist, that saves loading spots already visited up to time $t$ and forbids the ant to visit them againbefore $n$ iterations (a tour) have been completed. When atour is completed, the hlist is used to compute the ant's currentsolution (i.e. the movement cost of the path followedby the ant). The hlist is then emptied and the ant is free tochoose again.

\section{A. Hazard Intensity}

The quantify the hazard of a location, it introduces a novel metric called hazard intensity, whichis based on the observation that for an internal user, onemay feel more hazardous threat when getting closer toemergencies, and would feel safer when getting closer to exits.Inspired by the observation, any functioncan be used to represent the hazardous threats, as long as itis reversely proportional to the Euclidean distance betweenthe emergencies. Accordingly, we define a metric, hazardintensitywhich quantifies the hazardous threat atpoint $\mathrm{p}$ as follows:

$$
\overrightarrow{\mathrm{I}}(\mathrm{P})=\sum_{\mathrm{j}=1}^{\mathrm{m}} \mathrm{w}_{\mathrm{j}} \frac{\overrightarrow{\mathrm{PP}_{\mathrm{d}}^{\mathrm{j}}}}{\overrightarrow{\mathrm{PP}_{\mathrm{d}}^{\mathrm{j}^{3}}}}-\sum_{\mathrm{i}=1}^{\mathrm{n}} \mathrm{w}_{\mathrm{i}} \frac{\overrightarrow{\mathrm{PP}_{\mathrm{e}}^{\mathrm{i}}}}{\overrightarrow{\mathrm{PP}_{\mathrm{e}}^{\mathrm{i}}}} \ldots
$$

Obviously, the hazard intensity satisfies superpositionprinciple. In other words, the hazard intensity at point pinduced by multiple emergencies and exits is the sum ofthe responses that would have been caused by each stimulusseparately.

\section{B. Hazard Potential}

The hazard intensity reflects only aninstant feeling of the user, which is not enough to quantifythe hazard of a single point. To this end, it introduces afunction called hazardous potential $\Phi(\mathrm{p})$, which represents thetotal hazardous intensity one user has starting from infinityand ending at point $\mathrm{p}$. We choose infinity as a commonreference point to evaluate the hazard potential in differentspaces. Therefore, we define the hazard potential $\Phi(\mathrm{p})$ ofpoint $\mathrm{p}$ as follows:

$$
\Phi(\mathrm{p})=\int_{\infty}^{\mathrm{p}} \overrightarrow{\mathrm{I}}(\mathrm{l}) \mathrm{dl} \ldots . .
$$

The hazard potential describes the cumulative hazardintensity that the user should take when moving from pto infinity. Accordingly, the difference of hazard potential $\Phi$ (p)- $\Phi$ (q) between points $p$ and q can well measure whichpoint is more dangerous to the user. Specifically, a positivevalue of the difference of hazard potential indicates a higherchance of the user at $\mathrm{p}$ to be harmed than at $\mathrm{q}$. Therefore,the hazard potential can be used as a tool to quantitativelymeasure the amount of hazard of a single point.

\section{Hazard Potential Field}

The definition of single point hazard, now able to define the hazard potential field in a continuousspace, which plays an essential role in finding the safestpaths for trapped users.Based on Equ. (1) and (2), a hazard potential field $\Phi$ in acontinuous space $\mathcal{R}$ satisfies

$$
\nabla \Phi(\mathrm{p})=\mathrm{I}(\mathrm{p}) \ldots \ldots
$$

According to Gauss's Law, the potential field $\Phi$ satisfiesLaplace's equation 


$$
\nabla \mathrm{I}(\mathrm{p})=\nabla^{2} \Phi=0
$$

Note that the function satisfying Laplace's equation is calledharmonic function and has the mean value property.

D. Mean Value Property

$B(p, r)$ is a ball with the centerat point $p$ and the radius of $r$ in the open space $R$, thenthe value of $\Phi(p)$ at the center of the ball is given by theaverage value of $\Phi$ on the surface of the ball. In addition, $\Phi(p)$ is also equal to the average value of $\Phi$ in the interiorof the ball.

$$
\Phi(\mathrm{p})=\frac{1}{|\mathrm{~B}(\mathrm{p}, \mathrm{r})|} \int_{\mathrm{B}(\mathrm{p}, \mathrm{r})} \Phi \mathrm{d} \delta \ldots .
$$

$\mathrm{B}(\mathrm{p}, \mathrm{r})$ where is the volume of the ball in $\mathrm{R}$ and $\delta$ is the surface measure.

\section{E. Path Hazard Metric and the Safest Path}

The interested in finding the safest path from an arbitrarypoint $p \in P_{n}$ to an appropriate $\operatorname{exitp}_{\mathrm{e}}^{\mathrm{j}} \in \mathrm{P}_{\mathrm{e}}$. The primarychallenge of such problem is to choose the safest path amonghuge amounts of paths, which start from $p$ and end at $\mathrm{p}_{\mathrm{e}}^{\mathrm{j}}$.So in the first step, we have to design a path hazard metric toquantify the hazard of a path. As we discussed before, thelocation of point $p$ is more harmful to the user than that ofpoint $q$ if $\Phi(p)>\Phi(q)$. Intuitively, the hazard of a pathcan be quantitatively measured as the maximum hazardpotential of the points on the path. Therefore, we can definethe hazard of a path $\mathrm{C}$ as

$$
\mathrm{D}(\mathrm{C})=\max \{\Phi(\mathrm{p}) \mid, \mathrm{p} \in \mathrm{C})
$$

the objective is to find the safest path $\mathrm{C}^{*}$ from point pto pointp $\mathrm{p}_{\mathrm{e}}^{\mathrm{j}}$, such that the maximum hazard along the pathis minimum, i.e.,

$$
\mathrm{C}^{*}=\arg \min _{\mathrm{C}} \mathrm{D}(\mathrm{C}) \ldots .(7)
$$

Although we have this guideline to select the safest pathamong all possible paths, we still face several problems toimplement it in discrete WSNs where many constrains areneeded to be considered.

F. Algorithm Implementation

The discrete counterpart of hazard potential field in continuousdomains. Then propose an iterative method toestablish the hazard potential field by sensor readings in afully distributed manner. Based on the established hazardpotential field, we next propose a path selection methodand theoretically prove that the selected paths guarantee.

Step 1: Hazard Potential Field in Sensor Networks. The sensor network is then modeled as an undirected graph G (V, $\mathrm{E}$ ), where $\mathrm{V}$ is the set of vertices that represent thesensornodes, and $\mathrm{E}$ denotes the set of edges that represent thecommunication links between sensor nodes.

Step 2: hazard potential functionF(v) of a sensor node v satisfies

$$
F(v)=\frac{1}{|N(v)|} \sum_{u \in N(v)} F(v), v \in V_{n}
$$

where $\mathrm{N}(\mathrm{v})$ is the set of neighbor nodes of node $\mathrm{v}$ andjN(v)j is the cardinality of $\mathrm{N}(\mathrm{v})$.

Step 3: Iterative Hazard Potential Field Establishment, be more concrete, when there is no emergency,each node $v \in$ $\mathrm{Vn}$ is assigned a hazard potential value as 0 , while each sensor $\mathrm{v} \in \mathrm{Ve}$ is assigned a negative hazardpotential value reversely proportional to its capability. When the emergency happens, each sensor $\mathrm{v} \in \mathrm{Vd}$ will setits hazard potential value with a positive value proportionalto the hazard level of its reading.

Step 4: Dirichlet boundary condition, When the hazard potentialfunction $F(v)$ of $v \in V d$ [ $V e$ is fixed, every sensor $v \in$ Vnconducts the iteration as follows:

$$
\mathrm{F}^{(\mathrm{k}+1)}(\mathrm{v}) \leftarrow \frac{1}{|\mathrm{~N}(\mathrm{v})|} \sum_{\mathrm{u} \in \mathrm{N}(\mathrm{v})} \mathrm{F}^{\mathrm{k}}(\mathrm{v}), \quad \mathrm{v} \in \mathrm{v}_{\mathrm{n}}
$$

Dirichlet boundary condition, this iterativeprocess will finally converge if the hazard potential.

Step 5: Safest Paths Identification,established hazard potential field in the sensor network, it is straightforward to select the safest paths amongall possible paths that link the internal users and safe exits. 


\section{EXPERIMENTAL RESULTS}

NS2: The Network Simulator (ns2) is a discrete event driven simulator developed at UC Berkeley. We are using Network Simulator NS2 for simulations of protocols. It provides substantial support for simulation of TCP, routing and multicast protocols over wired and wireless networks. Ns-2 code is written either in C++ and OTCL and is kept in a separate file that is executed by OTCL interpreter, thus generating an output file for NAM (Network animator). It then plots the nodes in a position defined by the code script and exhibits the output of the nodes communicating with each other.

Congestion distribution. We measure a node's congestion by the number of scheduled paths the node involved in, where the scheduled paths refer to the paths generated by a specific navigation algorithm (i.e., CANS, SG or RM in our simulations).

Path stretch. the average/maximum stretch results of CANS, SG and RM methods for each network. It can be seen that CANS have an average stretch very close to 1 in all cases, the lowest among the three schemes. This means CANS has the highest navigation path planning efficiency, as the stretch is an indicator of navigation overhead. For example, an average stretch factor of 1.68 in RM indicates its overhead of 68 percent on the basis of the shortest path.

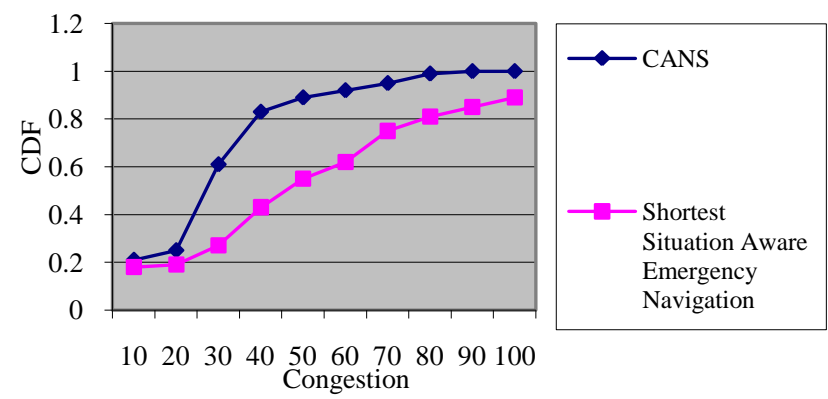

Fig 1. Comparison of proposed Congestion distribution.

Fig. 1 showscomparison of existing and proposed system different congestion value with CDF. The proposed system has only low value of CDF.

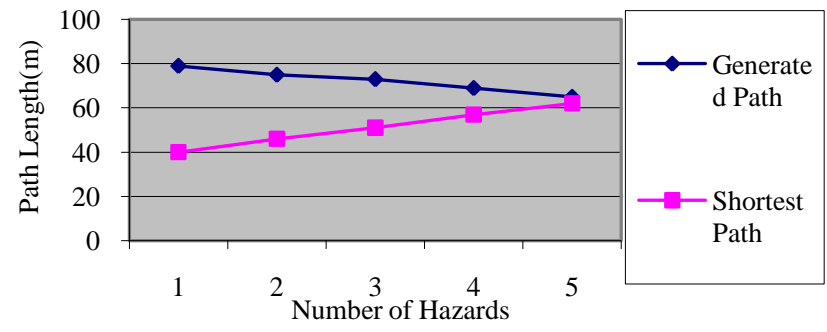

Fig 2. Path length versus number of hazards

Fig. 2 shows comparison of existing and proposed system number of hazard node vs path length in meter. The proposed system has shortest path exist.

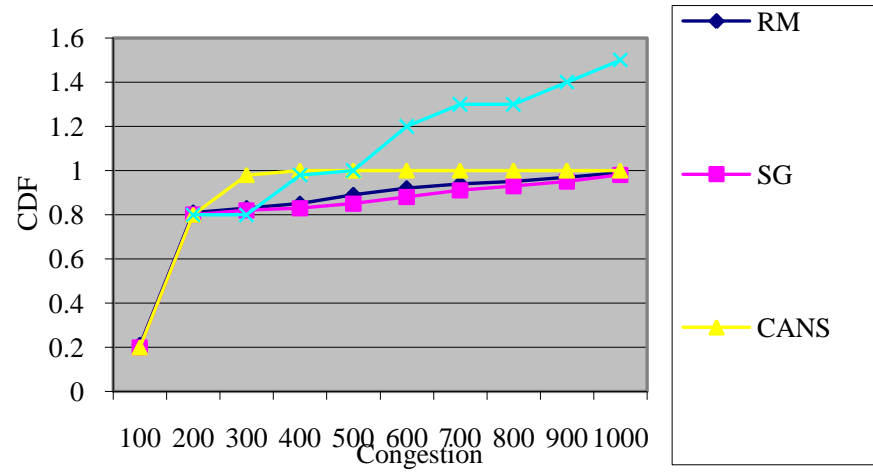

Fig 3. Comparison of proposed Congestion 
Fig. 3 shows comparison of existing and proposed system different congestion value in the network path vs CDF. The proposed system has low CDF.

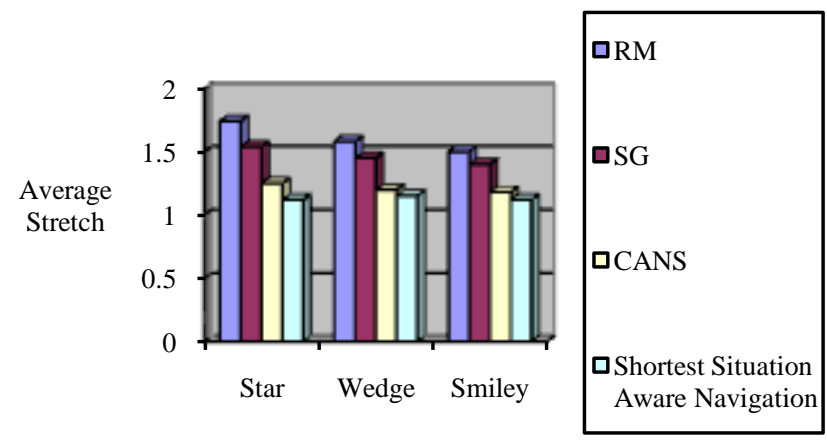

Fig 4. Comparison of proposed Average/maximum path stretch

Fig. 4 shows comparison of average stretch path existing and proposed.

\section{CONCLUSION}

The shortest path algorithm exceeds the ACOwhich is attributed to not yet been implemented evaporationof the pheromone, the pheromone amount in nature mayto remain a few hours to several months depending ondifferent aspects, such as ant species, soil type, causinga minor influence on the effect of evaporation in the processof finding the shortest path. Due to the long persistence ofpheromone, it is difficult for the ants to "forget" a path thathas a high level of pheromone but have found a way evens short. Keep in mind that if this behaviour is transferredto the computer to design a search algorithm sometimes itcan converge quickly to the local optimum. The proposed system show the results of the trial are presented. shortest situation-aware emergencynavigation by considering a more general and practicalproblem, where emergencies of different hazard levelsand exits with different evacuation capabilities may coexist. The first model the situation-aware emergency navigationproblem and formally define the safety of a navigation path. The propose a fully distributed algorithm to provideusers the safest navigation paths, as well as an acceleratedversion that can significantly boost up the speed of thenavigation.

\section{REFERENCES}

[1] Y. Song, B. Wang, Z. Shi, K. Pattipati, and S. Gupta, "Distributed algorithms for energy-efficient even self-deployment in mobile sensor networks,” IEEE Trans. Mobile Comput., vol. 13, no. 5, pp. 1035-1047, May 2014.

[2] C. Fischer and H. Gellersen, "Location and navigation support for emergency responders: A survey," IEEE Pervasive Comput., vol. 9, no. 1, pp. 38-47, Jan.-Mar. 2010.

[3] J. Wang, Z. Li, M. Li, Y. Liu, and Z. Yang, "Sensor network navigation without locations," IEEE Trans. Parallel Distrib. Syst., vol. 24, no. 7, pp. 1436-1446, July 2013.

[4] Q. Li, M. De Rosa, and D. Rus, "Distributed algorithms for guiding navigation across a sensor network," in Proc. 9th Annu. Int. Conf. Mobile Comput. Netw., 2003, pp. 313-325.

[5] E. Xu, Z. Ding, and S. Dasgupta, "Target tracking and mobile sensor navigation in wireless sensor networks," IEEE Trans. Mobile Comput., vol. 12, no. 1, pp. 177-186, Jan. 2013.

[6] C. Buragohain, D. Agrawal, and S. Suri, "Distributed navigation algorithms for sensor networks," in Proc. 25th IEEE Int. Conf. Comput. Commun., 2006, pp. 1-10.

[7] Y.-C. Tseng, M.-S. Pan, and Y.-Y. Tsai, "Wireless sensor networks for emergency navigation," Computer, vol. 39, no. 7, pp. 55-62, July 2006,

[8] M. Li, Y. Liu, J. Wang, and Z. Yang, "Sensor network navigation without locations," in Proc. 28th IEEE Int. Conf. Comput. Commun., 2009, pp. 2419-2427.

[9] Q. Xiao, B. Xiao, J. Luo, and G. Liu, "Reliable navigation of mobile sensors in wireless sensor networks without localization service," in Proc. 17th Int. Conf. Quality Service, 2009, pp. 1-9.

[10] A. Zhan, F. Wu, and G. Chen, "SOS: A safe, ordered, and speedy emergency navigation algorithm in wireless sensor networks," in Proc. 20th Int. Conf. Comput. Commun. Netw., 2011, pp. 1-6.

[11] S. MacLean and S. Datta, "Reducing the positional error of connectivity- based positioning algorithms through cooperation between neighbors," IEEE Trans. Mobile Comput., vol. 13, no. 8, pp. 1868-1882, Aug. 2014.

[12] G. E. Jan, K. Y. Chang, and I. Parberry, “Optimal path planning for mobile robot navigation,” IEEE/ASME Trans. Mechatronics, vol. 13, no. 4, pp. 451-460, Aug. 2008.

[13] Z. Yao and K. Gupta, "Distributed roadmaps for robot navigation in sensor networks," IEEE Trans. Robot., vol. 27, no. 5, pp. 997-1004, Oct. 2011.

[14] Z. Zhang, Z. Li, D. Zhang, and J. Chen, "Path planning and navigation for mobile robots in a hybrid sensor network without prior location information," Int. J. Adv. Robot. Syst., vol. 10, no. 172, pp. 1-12, 2013.

[15] M. Kuderer, C. Sprunk, H. Kretzschmar, and W. Burgard, "Online generation of homotopically distinct navigation paths," in Proc. 31st IEEE Int. Conf. Robot. Autom., 2014, pp. 6462-6467. 


\section{BIOGRAPHIES}

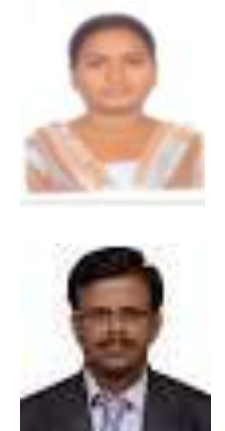

R. Bharathi, received my Bachelor Degree of Information Technology in Avinashilingam University at 2015, Started Master degree in SNS Collage of Engineering and research interest in area of networks (ie) Emergency Navigation Algorithm with WSN.

include Networks, Software Engineering and Wireless Networks.Email: ckalai2001@ gmail.com 